\title{
Grant Review Process
}

National Cancer Institute

\section{Source}

National Cancer Institute. Grant Review Process. NCI Thesaurus. Code C19475.

Official process of peer review for federal grants 